$\begin{array}{ll} & \text { Etnográfica } \\ \text { etnográfica } & \text { Revista do Centro em Rede de Investigação em }\end{array}$

Antropologia

vol. $24(3) \mid 2020$

Vol. 24 (3)

\title{
Déménager et ménager l'autorité : les unités domestiques du Haut-Atlas en question
}

Relocating and setting up authority: issues about domestic units of Hight-Atlas

\section{Pascal Mulet}

\section{(2) OpenEdition}

Journals

Édition électronique

URL : https://journals.openedition.org/etnografica/9427

DOI : $10.4000 /$ etnografica. 9427

ISSN : 2182-2891

\section{Éditeur}

Centro em Rede de Investigação em Antropologia

\section{Édition imprimée}

Date de publication : 1 octobre 2020

Pagination : 683-702

ISSN : 0873-6561

\section{Référence électronique}

Pascal Mulet, «Déménager et ménager l'autorité : les unités domestiques du Haut-Atlas en question », Etnográfica [En ligne], vol. 24 (3) | 2020, mis en ligne le 31 octobre 2020, consulté le 20 janvier 2022. URL : http://journals.openedition.org/etnografica/9427 ; DOI : https://doi.org/10.4000/etnografica. 9427

Etnográfica is licensed under a Creative Commons Attribution-NonCommercial 4.0 International License. 


\section{Déménager et ménager l'autorité : les unités domestiques du Haut-Atlas en question}

\section{Pascal Mulet}

Cet article vise à questionner les discours sur le gouvernement domestique dans le Haut-Atlas agropastoral au Maroc. Basé sur une étude ethnographique, il interroge les conditions de possibilité des discours sur la cohésion du groupe symbolisée par l'autorité du père. Il s'agit de mettre en lumière les pratiques par lesquelles cohésion et autorité sont actualisées, et de questionner les unités d'analyse utilisées en sciences sociales. La mise en regard des caractéristiques morphologiques de groupes domestiques ainsi que des pratiques et trajectoires de certains de leurs membres donne à voir comment la cohésion et l'autorité sont plus ou moins fortement soumises à tensions (et donc mises en péril). Il apparaît ainsi que le groupe (et l'autorité en son sein) s'actualise par un travail permanent des membres sur ses aspects sociaux, économiques et spatiaux, notamment par les pratiques matrimoniales et résidentielles ou encore les pratiques d'acquisition et de transmission de patrimoine. Cette approche permettra de questionner la « maison » comme objet de discours sur le gouvernement domestique dans le Haut-Atlas agropastoral au Maroc.

MOTS-CLÉS : agropastoralisme, économie, parenté, territoire, mobilité, maison.

Relocating and setting up authority: issues about domestic units of Hight-Atlas - This article attempts to question the speeches about domestic government in agropastoral Hight-Atlas in Morocco. Based on an ethnographical research, the article questions the conditions of possibility of speeches about group cohesion symbolized by father's authority. It aims to shed light on practices by which cohesion and authority are implemented, and to question units of analysis used by researchers. Comparison of domestic groups' morphological characteristics or member's practices and trajectories leads to observe that cohesion and authority are subject to tensions (and are therefore endangered). Doing so, one can see that a group (and the authority within it) is actualized by its membres' permanent work on its social, economic and spatial characteristics, such as for instance matrimonial and residential practices, or acquisition and devolution of inheritance. This approach allows to question the "house" as an object of speeches about domestic government, within agropastoral Hight-Atlas in Morocco.

KEYWORDS: agropastoralism, economy, kinship, territory, mobility, house.

MULET, Pascal (pmulet@laposte.net) - Laboratoire Temos, CNRS, Universités d'Angers, France. 
«SI LA MAISON ÉTAIT UN ÉTAT, MON PÈRE EN SERAIT LE PRÉSIDENT ». C'est par cette image qu'au début des années 2010 dans le Haut-Atlas au Maroc, un jeune homme récemment marié m'expliquait l'organisation et le fonctionnement de sa "maison ». Dans cette région agro-pastorale du Maghreb, milieu d'une économie largement organisée dans le cadre domestique et des relations familiales, il disait ainsi à l'ethnographe de citoyenneté française l'existence d'une institution (la maison) au sein de laquelle s'exerce l'autorité légitime d'un homme (son père) sur les autres membres du groupe (à la manière d'un président). Un autre me disait être l'équivalent du « roi » dans la sienne, un autre encore se désignait comme étant « le patron » à propos de son groupe domestique tourné vers l'élevage d'ovins et de caprins. Mobilisant des analogies différentes, ces hommes de la région berbérophone où j'ai mené une étude de terrain entre 2011 et 2014 donnent à entendre des discours visant à expliciter les logiques de gouvernement d'unités domestiques définies. ${ }^{1}$ Ils évoquent en effet l'autorité d'un homme et, ainsi, l'unité économique et sociale au sein de laquelle elle s'exerce : mode de gouvernement et unité se caractérisent mutuellement. De façon moins imagée, d'autres personnes m'expliquaient les pratiques et décisions économiques domestiques à partir de schèmes similaires.

Localement, l'unité cohabitante au sein de la maison est, selon les discours indigènes, également une unité politique dirigée par le père, formée autour de l'exploitation du patrimoine qu'il détient (propriété privée et droits d'usage). Les filles sont amenées à la quitter au moment de leur mariage dans le cadre du principe de virilocalité (j'ai pu entendre dire d'une fille ou d'une sœur mariée qu'elle est «partie à sa maison »[tdda s jgua-ns]). Les fils sont amenés à la quitter au moment appelé « division » [tibdit $i$, pratique de la dévolution des biens lors de laquelle un ou plusieurs fils quittent la maison et le groupe avec une partie du patrimoine (dont une maison habitable), que ce partage soit légalement notifié ou pas. ${ }^{2}$ À partir de ce moment, il est dit d'un fils qu'il « s'est autonomisé, s'est mis à l'écart » [isti]. Ainsi, selon ce modèle, division et décohabitation procèdent d'un même moment lors duquel émerge une nouvelle unité politique, économique et résidentielle. C’est généralement lors de la division que sont distinguées les parts de patrimoine destinées à chacune des filles, qu'elles soient mariées ou non. Localement, la virilocalité est associée à

I J'ai mené cette étude ethnographique dans le cadre de ma thèse de doctorat (2010-2015) à l'Ecole Normale Supérieure (Paris). Elle a notamment consisté en une installation entre 2011 et 2013 dans un village du Haut-Atlas, à partir duquel j'ai circulé au sein de divers réseaux, au Maroc et en Europe (tourisme et humanitaire). Je suis par la suite revenu plusieurs fois au village. Les propos rapportés ici ont été tenus en chleuh, la langue berbère locale, et traduits par moi-même.

2 Le partage pouvait se faire sous le regard de témoins villageois. Selon les enquêtés la parole de tels témoins fonctionnait comme preuve importante en cas de conflit porté au tribunal. 
un principe de « transmission sélective » en ligne masculine ${ }^{3}$ ainsi qu'à un système légal (reprenant les règles coraniques) de dévolution des biens octroyant aux filles une part d'héritage équivalente à la moitié de celles destinées au fils («Une part pour les fils et une demi-part pour les filles », m’expliquaient plusieurs enquêtés).

Les discours indigènes sur le gouvernement de la maison semblent confirmer les théories anthropologiques sur l'organisation tribale dans la région ainsi que les autres modèles savants de la «communauté domestique » développés à propos des milieux ruraux de diverses régions du monde, notamment en France. Dans leurs différents développements et formulations, ces modèles postulent l'existence de telles unités socio-économiques délimitées et cohérentes ainsi que de l'autorité masculine qui s'exerce en leur sein. Cet article vise au contraire à en montrer la contingence. Par la mise en regard de cas ethnographiques, je propose d'analyser les conditions de possibilité de la cohésion des groupes domestiques et de l'autorité en leur sein, et donc de tels discours sur le gouvernement de la maison. La notion d'exploitation agro-pastorale, configuration à la fois sociale, économique et spatiale, permet d'interroger les unités domestiques locales en rupture avec l'idéal d'unicité et d'homogénéité de la communauté domestique. Je montrerai comment l'exploitation est mise en forme et actualisée par un travail permanent de ses membres sur ses aspects sociaux, économiques et spatiaux, notamment dans le cadre des pratiques matrimoniales et résidentielles, ou encore des pratiques d'acquisition et de transmission de patrimoine. C'est dans le cadre d'un tel travail que des hommes tenaient auprès de moi des discours qui semblent confirmer les représentations savantes développées à propos des régions rurales, notamment du Maghreb.

\section{QUELLES UNITÉS D'ANALYSE?}

Le modèle de la communauté domestique a une longue histoire en sociologie et en anthropologie des milieux ruraux. J'en présenterai quelques traits saillants avant de proposer une approche du domestique dans le Haut-Atlas axée sur la notion d'exploitation.

\section{La communauté domestique en anthropologie rurale : unité et autorité}

La communauté domestique est un élément d'une communauté plus large : la «communauté villageoise » de la sociologie rurale française du Groupe de Sociologie Rurale (Jollivet et Mendras 1971 ; Mendras 1976), ou encore

3 C'est-à-dire un principe selon lequel, en théorie, seuls les fils disposent de « la faculté de fonder [leur] propre groupe domestique, éventuellement de continuer celui des parents, de succéder au sens originel du terme (venir à la place de) » (Augustins 1998). 
la «tribu» de l'anthropologie du Maghreb (Berque 1954 ; Jamous 1981 ; Gellner 2003 ; Mahdi 2009). Lidéal de la communauté comme "un tout » (Redfield 1989) repose sur un paradigme développé au cours du XIX ${ }^{e}$ siècle (Maine 1889 ; Tönnies 1944) : en marge de la société, milieu de l'échange par contrat d'individus dotés d'une rationalité relevant de l'utilitarisme benthamien, les communautés constituent des groupes organiques dotés de leur volonté propre. C'est un tel cadre conceptuel qui est mobilisé pour décrire, en France comme au Maroc, des communautés rurales occupant et travaillant des territoires délimités, et structurés selon des règles « traditionnelles » (Mahdi 1999 : 31 ; Billaud 2009). Proposant une vue synoptique des groupes sociaux et de leur territoire, ces analyses font de la communauté domestique la plus petite unité sociale et territoriale observable, dernière sous-division d'une communauté plus large. ${ }^{4}$ Dans cette perspective, la communauté domestique est plus qu'une unité d'analyse et paraît être une unité existant de fait, un en-soi : une unicité cristallisée et figée, la fusion des personnes, de l'habitat et du territoire exploité. Selon Henri Mendras (1976), le terme de groupe domestique « met l'accent sur l'ensemble des gens vivant dans la maison et évoque à la fois l'économie domestique qui répond au besoin du groupe, et le domaine qui le fait vivre ». La communauté domestique constitue une totalité au même titre que la communauté plus grande dans laquelle elle s'inscrit.

Dans ce modèle de la communauté domestique, ces unités sont définies à partir de divers éléments. À propos des groupes domestiques des sociétés paysannes, Henri Mendras (1976) fait de la cohabitation la caractéristique première des groupes domestiques («au même feu et au même lieu »), avant d'en questionner les types de gouvernement selon les régions. À propos d'éleveurs nomades au Maroc, Hassan Rachik (2000 : 30) reprend la notion indigène de doukhan (fumée), constitué par « les personnes qui ont une cuisine (une «fumée ») commune. Il s'agit d'une unité de consommation qui peut comprendre plusieurs familles conjugales habitant dans plusieurs tentes, mais groupées sous l'autorité d'un même chef ». Le type d'autorité qui s'exerce au sein des unités domestiques est une de leurs caractéristiques. L'autorité d'un homme sur les autres membres du groupe peut être définie comme étant « patriarcale », pater désignant « tout homme qui ne dépendait d'aucun autre et qui avait autorité sur une famille et un domaine »(Coulanges 1864, cité par Delphy 2000). ${ }^{5}$ Selon Christine Delphy (2000 : 142), au XIX siècle en

4 C’est également la représentation proposée par Marx des sociétés rurales : « La parcelle, le paysan et sa famille ; à côté une autre parcelle, un autre paysan et une autre famille. Un certain nombre de ces familles forment un village, et un certain nombre de villages un département » Marx (1963) cité dans Jollivet (1974).

5 Cette perspective est proche de celle proposée par Aristote (1962:32), selon lequel l'économie domestique est composée de trois parties, ou trois relations : maître et esclave, époux et épouse, père et enfant. 
Europe, « le mot [patriarcat] connote de petites communautés agricoles composées d'unités familiales de production, chacune sous la houlette de son ancêtre, la vie communautaire était réglée par la réunion des ancêtres - des chefs de famille ». Le patriarcat relève donc du modèle de la communauté domestique : d'une part en tant que caractéristique structurale d'une société dite patriarcale ; et, d'autre part, en tant que mode de gouvernement - et donc caractéristique - des groupes domestiques qui la composent. Dans le Maghreb rural caractérisé en sciences sociales par l'agnation comme base des structures segmentaires (Durkheim 2007 ; Gellner 2003), ou encore par la visibilité de la domination masculine (Bourdieu 1998), l'autorité domestique est masculine par principe structural. ${ }^{6}$ Ainsi, selon Robert Montagne, un des principaux théoriciens de l'organisation tribale au Maroc, le groupe familial, patriarcal et agnatique, qui habite une partie du village est « l'unité la plus petite, mais aussi la plus vivante, qui contribue à former des groupes sociaux plus vastes en pays berbère » (Montagne 1930: 43 ; voir également Jamous 1981 : 36). ${ }^{7}$ En ce sens, les limites et les formes de ces unités sont définies par les limites et les formes du pouvoir qui s'y exerce dans le cadre de structures sociales déterminées.

\section{Questionner les pratiques : «maison » et « exploitation agropastorale»}

Les analyses que Pierre Bourdieu a consacrées à la maison pyrénéenne (Bourdieu 1962) et à la maison kabyle (Bourdieu 2000 [1972]) comme objets et cadres de stratégies visant à leur perpétuation permettent d'envisager ces unités agropastorales comme produits de la pratique. L'intérêt pour les dynamiques propres à la maison (Augustins 1977), « une configuration spatiale, économique et sociale spécifique »(Chiva et Goy 1981 : 11), permet de rompre avec l'idéal d'homogénéité et de stabilité qui caractérise les théories de la communauté. Cependant, la perspective ouverte par Bourdieu conserve l'idéal d'unité domestique cohérente formant un tout à la fois social, spatial, économique (Bender 1967) et politique. ${ }^{8}$ Il présente une communauté relativement autonome, caractérisée par une forme spécifique d'autorité et traversée par ses propres tensions, au sein de la maison, bâtiment qui constitue (avec le patrimoine foncier qui y est attaché) l'unité de lieu de toutes les pratiques

6 Sur la proximité entre la notion d'habitus, proposée par Pierre Bourdieu à propos des sociétés kabyles, et les représentations structuralistes, voir Hammoudi (2007).

7 Plus tard, il revient sur l'importance de celle-ci du fait de ses attaches avec les terres cultivables : «C'est, en fin de compte, à la famille patriarcale qu'il faut en venir pour expliquer toutes les formes de la vie sociale et politique de la tribu» (Montagne $1953: 21$ ).

8 La définition par Bourdieu de la maison pyrénéenne comme étant « à la fois lignage et patrimoine » (Bourdieu 1962) fait écho à celle de la maison par Claude Lévi-Strauss (1979 : 157). Cependant, leurs approches diffèrent, car Lévi-Strauss considère les doubles appartenances et autres formes d'antagonismes comme étant constitutives de la maison comme unité sociale. 
domestiques. ${ }^{9}$ D'autres approches donnent à voir la diversité des lieux de l'économie rurale (Fontaine 1994 et 2005) et des territoires des « configurations familiales » rurales (Rosental 1999), ou encore la manière dont l'organisation de l'économie paysanne (Tchayanov 1990) consiste à placer la «main-d'œuvre familiale » sur le domaine du groupe et sur le marché du travail. L'observation des pratiques domestiques sans présupposer l'unité de lieu qui en serait le théâtre permet d'analyser comment se constituent, dans la même dynamique, les groupes domestiques et les formes d'autorité en leur sein. Il s'agit ainsi de considérer comment émergent et s'actualisent et se soutiennent mutuellement des configurations sociales, des configurations spatiales et des configurations économiques, produits des interactions entre les individus et des jeux d'équilibre des forces et des tensions (Elias 2003 [1970] ; Araos 2019).

L'analyse de la parenté comme produit de pratiques économiques (Carsten 1995 ; Weber 2005 ; Sahlins 2011 ) permet de saisir comment prennent forme les groupes domestiques ainsi que les rapports de pouvoir en leur sein. ${ }^{10}$ Dans cette perspective, la «maisonnée » (Weber 2002) est un groupe domestique constitué autour de « causes communes » (Gollac 2003) et dans le cadre de pratiques de mutualisation des ressources (Perrin-Heredia 2011). L'approche par les pratiques économiques permet de voir comment les membres d'une maisonnée circulent entre plusieurs sphères sociales (au sein desquelles ils acquièrent ressources, capitaux et statut social), habitent éventuellement plusieurs logements, et peuvent appartenir à plusieurs groupes, se trouver « au croisement de plusieurs nous» (Weber 2005 : 16). Cette approche permet de saisir les configurations sociales et économiques qui émergent, se transforment, s'imbriquent et disparaissent dans la pratique. L'analyse des mobilités et des pratiques résidentielles donne également à voir comment le travail sur l'espace domestique participe à la cohésion des groupes domestiques et à la gestion des tensions en leur sein. Considérer la pluri-localité du domestique permet d'observer les jeux sur la distance entre maisons lointaines, voisines ou

9 La maison béarnaise et la maison kabyle analysées par Bourdieu présentent de nombreuses similitudes malgré les différences entre les deux régions en termes d'organisation sociale et de dévolution des biens. Dans le chapitre de l'Esquisse d'Une Théorie de la Pratique consacré à la parenté (2000 [1972] : 62 et suivantes), Bourdieu aborde la question de l'autorité dans «l'unité domestique » (aussi appelée «maison », « famille», « famille indivise », « lignée », « lignage », « exploitation familiale ») qui s'inscrit au sein d'unités politiques plus larges, comme le clan ou la tribu. S'il questionne « la structure des rapports de pouvoir domestique », « la structure des rapports de force et d'autorité » et les stratégies divergentes qui peuvent mener à la rupture, c'est à partir de l'idée d'un groupe fermé, d'une unité de lieu et d'une autorité domestique d'abord détenue par (ou « qui incombe à») un homme (le «patriarche » ou celui « qui a de longue date préparé sa succession»).

10 Claude Meillassoux (1975) analyse les logiques de la construction de l'autorité de l'aîné dans « la communauté domestique » à partir des intérêts en jeu dans les pratiques économiques et matrimoniales. Dans cette perspective marxienne, la forme du groupe domestique et l'autorité en son sein ne sont pas données ni expliquées l'une par l'autre, mais bien construites dans la pratique. 
au sein même de l'habitat. Dans cette perspective, une « configuration résidentielle » (Pfirsch 2009 ; Motta 2014 ; Araos 2016) est constituée par les pratiques de résidence, de bâti, d'aménagement, de limitation, ou de circulation de ses membres.

Dans le Haut-Atlas où j'ai mené mon étude de terrain, la «maison » semble a priori être une unité fondamentale pour comprendre l'organisation sociale. Cependant, afin de fonder l'analyse sur l'observation des pratiques (économiques, relationnelles, résidentielles, etc.) en considérant la pluri-localité du domestique, j'utiliserai plutôt la notion d'exploitation agropastorale. Dans le contexte local, l'exploitation n'est pas une catégorie administrative ou légale, elle n'est pas une personne morale, et le terme n'est pas la traduction d'un terme indigène. L'exploitation est d'abord appréhendée dans sa dimension économique : elle est une unité non exclusive de production et de consommation formée autour d'un patrimoine ainsi mis en valeur. Dans la perspective que je propose, l'exploitation est une forme de maisonnée si l'on s'intéresse aux pratiques économiques. Elle est une configuration résidentielle si l'on s'intéresse aux pratiques d'habitat et de bâti. Elle est également une configuration territoriale (Mulet 2018) si l'on s'intéresse aux pratiques de production agro-pastorale (acquisition de terres, mobilités entre les lieux de l'exploitation, etc.). Cette approche permet d'interroger les particularités de la maison comme unité de corésidence (élément d'un territoire domestique plus vaste).

Considérer ensemble les jeux et arrangements sur les aspects sociaux, économiques et spatiaux des exploitations permettra d'interroger les conditions de possibilité de la cohésion des groupes et de l'autorité domestique. Les deux cas présentés permettront d'analyser de telles pratiques. ${ }^{11}$ Le premier, celui des Hasima, donne à voir une autorité et une cohésion apparemment acceptées par le fils aîné, pourvoyeur de ressources monétaires. Le second, celui des Utena, permettra de présenter les tensions qui apparaissent au sein du groupe et les modes de gestion de celles-ci. L'analyse des pratiques économiques de ces deux groupes - au sein desquels certains hommes détiennent un pouvoir du discours sur ces pratiques auprès de l'ethnographe (voir l'encadré ci-dessous) - constituera le fil de cette comparaison des exploitations, considérées dans un premier temps comme configurations sociales puis, dans un second temps, comme configurations spatiales. Dans la pratique, les jeux des membres sur les aspects sociaux, économiques et spatiaux de leur exploitation sont éminemment liés et interdépendants.

11 Par souci d'anonymat, les noms de personnes ont été changés. Afin qu'elles ne soient pas reconnaissables dans leurs milieux d'interconnaissance, j’ai également modifié des éléments de leur présentation, en veillant à ne pas modifier le sens de mon analyse des cas observés. 
FILS ET FILLES DANS LES FRATRIES, PRATIQUES MATRIMONIALES ET CONSTITUTION DES GROUPES

Considérer l'exploitation comme configuration sociale permet d'interroger les logiques de la constitution d'un tel groupe de production et de consommation, qui est également un groupe politique. Je propose de l'observer au prisme de la question de la division du travail. La prise en compte des caractéristiques démographiques des fratries et des pratiques matrimoniales donne à voir les processus d'inclusion et d'exclusion, la diversité des statuts au sein des exploitations ainsi que le champ des possibles en termes de poly-activité économique. Je questionnerai les pratiques et les relations effectives entre membres afin de mettre en lumière certaines des conditions démographiques et économiques de ces rapports de pouvoir dans la pratique.

\section{Un aîné et son jeune frère}

Said Hasima, un jeune homme né en 1983, habitait la maison de ses parents et participait à cette économie poly-active par le travail salarié. S'il n'était pas le premier enfant de la fratrie, Said avait un statut d'aîné en tant que premier fils. Parmi les filles de la fratrie, sa sœur aînée avait quitté l'exploitation au moment de son mariage. Le seul frère de Said, cadet de la fratrie, était scolarisé dans l'école du village. Said pourvoyait en grande partie aux besoins monétaires du groupe, parmi lesquels ceux liés aux études, en ville, de sa sœur cadette. Mobilisant des réseaux de travail qui s'étendaient sur tout le royaume, il a régulièrement migré pour se faire employer sur des chantiers de construction. Il a ainsi acquis un savoir-faire, développé son capital social, s'est familiarisé avec la mobilité et s'est spécialisé. Il a ainsi pu évoluer socialement : mieux rémunéré, il se faisait employer directement par les chefs d'entreprises (plutôt que par des sous-traitants non-déclarés) avec lesquels il pouvait négocier son salaire (mensuel et non journalier). Par la suite, Said s'est fait employer localement, dans un autre domaine d'activité. C'est ainsi qu'il participait très activement à l'économie domestique, par le financement de dépenses courantes ou plus exceptionnelles comme par exemple certains travaux dans la maison.

Said tenait auprès de moi un discours très clair sur le gouvernement domestique, la place qu'y tenaient les différents membres et l'autorité qu'il reconnaissait à son père. C'est lui qui, après m'avoir indiqué que sa relation avec ses parents primait sur celle avec sa femme, avait eu recours à une métaphore politique : «Si la maison [jgua] était un État [dula], mon père en serait le président ». Ce discours va dans le sens des pratiques que j'ai pu observer au sein de cette maison. Alors que son père n'investissait pas dans le foncier agricole ou dans de nouvelles activités productives ou rémunératrices, Said tenait une position importante, si ce n'est centrale, dans cette configuration économique. Malgré cette position, le discours qu'il tenait auprès de moi, et surtout ses 
pratiques matrimoniale, résidentielle et économique, semblaient alors confirmer l'hypothèse patriarcale. Cependant, se pose la question de la définition et des limites de la «maison» dont parle Said. Une autre «maison» doit être prise en compte pour analyser les enjeux de gouvernement domestique : celle, quasiment voisine, des deux tantes et de la grand-mère paternelles de Said. Je présenterai d'abord l'exploitation des Utena.

\section{Situations ethnographiques et gouvernement domestique}

Alors que je me rendais régulièrement chez lui et avait de bonnes relations avec tous les habitants (femmes et hommes) de la maison, Said Hasima ne m'a, à aucun moment, présenté sa femme, ou présenté à elle. Après leur mariage, nous nous croisions souvent chez Said, mais nous n'avons quasiment pas discuté. Chez les Utena, les femmes (mère, fille, belles-filles) ne me parlaient quasiment jamais, ou peu lorsque je me rendais chez elles. Ces mises en œuvre spécifiques de la domination masculine ne doivent pas être généralisées à l'ensemble de mon terrain. L'analyse au cas par cas des situations concrètes de l'étude de terrain permet de saisir certaines formes que prend cette domination masculine. Par exemple, la mise en ouvre d'une forme de «patriarcat » est particulièrement explicite chez les Utena si l'on prend en compte l'effacement des fils d'Ahmed en sa présence et la priorité qu'avait ce dernier dans la relation à l'ethnographe.

\section{Une fratrie plus nombreuse et majoritairement masculine}

Ahmed Utena est le père d'une fratrie plus nombreuse et composée majoritairement de fils. Les Utena se distinguent également des Hasima par la diversité des activités et des sources de revenus de l'exploitation. Durant mon étude de terrain, le groupe n'avait pas divisé. L'aîné était salarié à proximité de la maison de son père après quelques années d'études. Il habitait avec sa femme et leurs enfants dans leur propre maison, construite sur des terres appartenant à Ahmed à proximité de la maison de ce dernier. Cette décohabitation, alors que ce dernier affirmait auprès de moi l'unité du groupe («On va toujours ensemble »[Nsul nman]) m'a longuement posé question. Elle semble être un mode de gestion des tensions au sein du groupe dont je présenterai les aînés, parmi lesquels une fille, mariée et habitant avec son mari dans la vallée.

Alors que l'aîné était employé localement, le deuxième et le troisième fils d'Ahmed - Brahim et Mustafa - semblaient avoir acquis dans la migration des ressources non négligeables et une aisance leur ouvrant la possibilité de se projeter dans ce type d'activité qu'ils valorisaient parfois auprès de moi. Brahim, né en 1980, était marié avec Sadia, originaire du village voisin et qui, depuis, vivait et travaillait dans la maison d'Ahmed Utena. Brahim me racontait avoir 
auparavant envisagé de se marier avec une femme alors qu'il était en migration, dans une ville que ses parents jugeaient «trop loin », motif de leur refus. «Alors je suis revenu », m’expliquait-il. Mustafa, né en 1982, migrait également. Lors d'un de ses séjours dans la maison de son père, il m'expliquait son ascension sociale dans la migration avant de m'expliquer « qu'ici il n'y a pas de travail, pas d'argent, il faut partir ». Quelques mois plus tard, à nouveau au village, il m'annonçait vouloir se consacrer aux activités agricoles au village et à une activité de commerce au marché de la vallée, auprès de son père. Il m'apprenait également son mariage avec une jeune femme du village que lui avaient présenté ses parents quelques temps auparavant. Il m'avait déjà parlé d'un projet de mariage :

«... ceux de la maison [Ayt jgua] m'ont dit : 'Il faut que tu te maries'

Ceux de la maison : ton père, Brahim...

Oui. Ils m'ont dit : 'Il faut que tu te maries.'

Pourquoi?

Ils m'ont dit: 'Il y a beaucoup de travail, il faut que tu te maries.' Je leur ai dit d'accord. C'est tout.

Parce que dans la maison, il y a Sadia [la femme de Brahim] ...

Sadia, c'est tout. » [entretien avec Mustafa Utena, 201 l]

Le mariage, lors duquel sa femme a intégré « la maison », a eu lieu quelques mois après. La veille de la cérémonie, alors que j'étais chez Ahmed Utena, l'aîné Yussef m'avait dit, sous forme de blague, de revenir tôt le lendemain afin de travailler aux préparatifs. Plus tard, son frère Brahim me disait que Yussef était « un invité » qui allait dormir chez lui ce soir. Il semble alors ne pas le considérer comme membre du groupe de «ceux de la maison», les «Ayt jgua», si l'on reprend le terme utilisé par Mustafa en entretien. ${ }^{12}$

Les pratiques matrimoniales des fils aînés d'Ahmed semblent confirmer l'hypothèse patriarcale, notamment en ce qui concerne le choix de l'épouse, amenée à habiter et travailler dans l'exploitation, dans ces cas où la virilocalité est effective. En entretien, Ahmed m'avait expliqué les logiques de ce choix matrimonial ( «Une femme bien, ce n'est pas le visage. C'est une femme qui travaille bien», notament), ainsi que le mode de fonctionnement d'une telle exploitation : les fils remettent la quasi-totalité des revenus de leurs travaux rémunérés à leur père, ce dernier ayant la charge des dépenses courantes et d'investissement de toute l'exploitation, c'est-à-dire le pouvoir de gestion

12 La définition de la «maison » semble différente de celle d'un parent des Utena qui m'expliquait, alors que je le questionnais sur les termes indigènes et sur le lien entre division et décohabitation, à propos de ceux-ci que : «C'est une seule jgua, puisque Yussef n’a pas divisé. Il fait partie de la maison d'Ahmed. Pour payer l'imam, ils comptent comme une seule maison». [journal de terrain, 2012]. 
du budget collectif et d'orientation de l'économie du groupe. Ainsi, m'expliquait-il, son fils Brahim avait acheté avec son autorisation le véhicule avec lequel il travaillait. Le bien était ainsi la propriété du groupe et chacun aurait droit à une part de sa valeur dans l'hypothèse d'une division du patrimoine :

«Brahim a acheté une voiture. Elle est à nous tous. Si on veut diviser, on compte tout, on divise tout. Comme ça. C'est comme ça notre système, on fait tous comme ça ». [entretien avec Ahmed Utena, 2013]

Dans la pratique, le système est plus complexe. La notion de propriété collective n'est pas toujours celle mise en avant par les fils d'Ahmed, même s'ils jouent parfois le jeu de l'indivision.

Dans le système de l'exploitation, ceux qui étudiaient étaient pris en charge financièrement par leur père ainsi que leurs frères disposant de revenus. Cette circulation monétaire donne à voir un modèle qui n'est plus centré sur le père, ainsi qu'une logique qui n'est pas celle de la mise en commun, mais de la relation entre individus. Émergent ainsi, au sein du groupe domestique, les enjeux du don et du contre-don et des statuts de donateurs et de donataires. En d'autres termes, on observe au sein de la maisonnée, groupe de coopération basé sur la mutualisation, des logiques de parentèle basées sur les échanges entre personnes (Weber 2002 ; Gollac 2003). La décohabitation de plusieurs membres de l'exploitation semble à la fois une manière de gérer les tensions et l'objet de nouvelles questions concernant la propriété, notamment la propriété foncière.

L'analyse de ces exploitations comme configurations sociales donne à voir la complexité de leurs formes ainsi que des statuts et des relations en leur sein, qui évoluent notamment dans le cadre des divers types de circulations de personnes, de biens ou de droits. Les caractéristiques morphologiques des fratries doivent être prises en compte pour saisir les enjeux de gouvernement domestique. Un grand nombre d'enfants, et notamment de garçons comme dans le cas des Utena, permet de disposer d'une force de travail importante pour accéder à de nombreux types de ressources. Dans le premier cas, par contre, l'aîné Said Hasima est le principal pourvoyeur de monnaie de l'exploitation dirigée par son père. La tension entre collectivisation et individualisation semble faible dans l'exploitation des Hasima. Dans le contexte local, les sœurs de Said Hasima sont amenées à quitter l'exploitation. Quant à son seul frère, alors enfant et scolarisé, il ne faisait pas obstacle au processus d'ascension sociale de l'aîné. Ce dernier peut alors entraîner l'exploitation dans ce processus débuté par la migration et en réinvestir les revenus dans la maison paternelle dont il a toutes les chances d'hériter en tant qu'aîné et au sein de laquelle les investissements réalisés participent de la construction, sur le long terme, de sa propre autorité. Les caractéristiques sociales des exploitations, cependant, 
ne se limitent pas à celles des fratries. Les mariages des fils et les choix des épouses relèvent d'enjeux démographiques de constitution des exploitations, qu'il s'agisse de garder un fils à proximité ou d'intégrer au groupe une bru qui sera amenée à cohabiter et à travailler dans la maison. Je reviendrai maintenant sur cette complexité en centrant l'analyse sur les configurations résidentielles et territoriales ainsi constituées.

\section{PRODUCTION DES TERRITOIRES DE LA PARENTÉ :}

\section{LIEUX DE POUVOIR}

Je traiterai des formes des exploitations et de l'autorité en leur sein par le biais de l'analyse de l'habitat et de la propriété foncière. Ce travail sur l'espace se fait en grande partie dans le cadre des pratiques de la dévolution des biens favorisant les fils par rapport aux filles. Le cas des Hasima donne à voir la mise en place d'une continuité agnatique au sein d'une maison. Le cas des Utena éclaire la gestion des forces de fusion et forces de fission (Bourdieu 1993) qui traversent le groupe.

\section{Bi-résidence et lignée masculine}

La valorisation par Said Hasima du mode de gouvernement patriarcal prend sens également par rapport au processus de construction de la lignée, que j’interrogerai à partir de l'observation des pratiques de dévolution des biens et d'habitat. L'histoire de la lignée de Said Hasima est celle d'une continuité en ligne masculine au sein de la maison d'habitation dans le cadre « d'arrangements » (Bessière 2010) sur les questions d'héritage et d'habitat qui permettent ce type de succession agnatique.

Lhsen, le père de Said, est l'aîné et unique garçon de sa fratrie. Du vivant de leur père, deux de ses sœurs, non mariées, ont fait construire une maison au village dans laquelle elles se sont installées avec l'enfant de l'une d'elles. Leur mère les y a rejointes après le décès de leur père. Les trois femmes habitaient et élevaient un petit troupeau dans cette maison. Cette configuration particulière peut être considérée comme un arrangement autour des normes de la division. Lhsen Hasima m'expliquait la manière dont avait été transmis le patrimoine foncier depuis son propre grand-père paternel. Au moment de cette division, le nombre de filles était égal au nombre de fils dans la fratrie du père de Lhsen. Le grand-père de Lhsen avait alors divisé le patrimoine transmis ainsi : chaque fils recevait dans un premier temps une partie équivalent à sa part plus la demipart d'une de ses sœurs. Par la suite, celles-ci réclamaient ou pas leur demi-part au frère qui en disposait. Selon Lhsen, la sœur de son père qui avait sa demipart chez ce dernier la lui a réclamée.

Le père de Lhsen n'a pas divisé avec ce dernier qui, successeur à la tête de l'exploitation dont il n'est pas l'unique héritier, me disait néanmoins avoir 
« donné » deux parcelles à ses sœurs. Il ne s'agit pas là d'une division, mais d'un transfert de droits d'usage destiné à assurer une certaine autonomie à la maison des femmes : une parcelle était valorisée comme jardin potager et l'autre comme champ de luzerne, destiné à l'alimentation du bétail. Le reste des champs était exploité en commun par les deux maisons, pour produire de l'orge et du maïs (alimentation du bétail) ainsi que des pommes de terre, notamment. Une telle production nécessite des travaux « masculins » (les labours, notamment), une force de travail parfois importante et l'emploi d'une main d'œuvre rémunérée, donc coûteuse en termes monétaires. Lhsen me disait payer tous les frais liés à la production agricole et apporter chaque semaine du marché un demi-kilo de viande à la maison de ses sœurs et de sa mère. « Il faut s'aider », m'expliquait-il. Si les revenus agricoles étaient partagés entre les deux maisons, chaque groupe de cohabitants gérait un budget autonome, composé à la fois de revenus agricoles et de revenus monétaires propres.

Ainsi, le cas des Hasima permet d'observer un type d'arrangement sur les éléments spatiaux, économiques et sociaux de l'exploitation. Alors qu'en théorie division et décohabitation sont associées, Lhsen Hasima et ses sœurs ont mise en place une forme d'indivision basée sur la bi-résidence dont les enjeux sont autant spatiaux qu'économiques, puisque les deux maisons - celle de l'aîné et celle des femmes - sont liées dans le cadre d'une partie de la production agricole et autonomes en tant qu'unités économiques basées sur la cohabitation d'apparentés. C'est dans ce contexte qu'est construite et actualisée l'autorité de Lhsen, reconnue et valorisée par son fils Said. L'analyse du gouvernement de la maison nécessite ici de considérer la distinction entre le groupe des cohabitants et le groupe des co-héritiers (Gollac 2005) pour saisir les formes et les limites de l'autorité du père et de l'aîné. La mise en regard de cette exploitation avec celles d'Ahmed Utena permettra de revenir sur les enjeux de la résidence dans la production d'une telle autorité et la gestion des tensions.

\section{Bi-résidence et tensions}

Unique héritier, Ahmed Utena a hérité d'un patrimoine foncier dont l'importance peut expliquer la renonciation de ses aînés à l'éloignement par la migration. Ce pouvoir qu'avait Ahmed de les garder auprès de lui était en tension avec le pouvoir qu'avaient ceux-ci de s'autonomiser par rapport à lui, et donc au groupe, dans le cadre de la migration. Cette tension se joue également à l'échelle du territoire du village. L'aîné de ses fils (Yussef) avait construit une maison sur des terres appartenant à son père, avec l'aide financière de son père et de certains de ses frères. Il y habitait avec sa femme et leurs enfants. Le couple y élevait quelques brebis. Par la suite Yussef avait entrepris de coûteux travaux d'aménagement de la maison. Cette décohabitation, tandis que Ahmed ne cessait de me dire que l'aîné et lui « [allaient] toujours ensemble », m'avait longuement dérouté. J'avais en effet appris, au village, que décohabitation 
et division constituaient un même moment lors duquel un fils s'autonomisait [isti] par rapport à son père. Concernant les Utena, cette décohabitation sans division et d'autres contradictions apparentes du même type sont liées aux tensions qui traversent le groupe : entre tendance à l'individualisation et tendance à la collectivisation, entre pouvoirs individuels et autorité du père sur l'ensemble du groupe.

Selon certains de leurs proches, la décohabitation de l'aîné était en partie liée aux fréquentes et violentes disputes entre sa mère et Tuda, son épouse, alors que les deux femmes cohabitaient et partageaient les travaux quotidiens. Il m'est apparu par la suite que l'argument de la « dispute des femmes » (effective ou potentielle) était un argument souvent avancé pour justifier une décohabitation (effective ou en projet). ${ }^{13}$ Selon cette hypothèse, les risques de rupture dans l'exploitation des Utena ont été minimisés par une détente des liens de pouvoir, elle-même rendue possible par la bi-résidence. Yussef et sa femme avaient acquis un lieu propre, un espace domestique qu'ils géraient et investissaient à leur guise. C'est même son propre budget que gérait Yussef, comme me l'expliquait son père alors que j'insistais pour comprendre la place de l'aîné dans l'exploitation :

« Pas de problème, maintenant, avec lui. On n’a pas divisé. Mais maintenant, lui il construit sa maison. Je vois qu'il construit sa maison, il a beaucoup de frais. Je lui laisse son salaire, il construit sa maison, il paye les ouvriers, mais ce qui lui reste, il me le donne. S'il m'en faut, il m'en donne, pas de problème ». [entretien avec Ahmed Utena, 2013]

La maison, instrument de l'autonomisation résidentielle, constitue aussi le cadre d'une autonomisation budgétaire au sein du groupe en indivision. Dans le cadre de son emploi, Brahim, le deuxième fils d'Ahmed, circulait entre la maison paternelle où vivaient sa femme et ses enfants et le petit appartement qu'il louait dans la ville du piémont, et dans lequel un de ses frères cadets vivait le temps de ses études. Mustafa, le troisième fils d'Ahmed, alors marié, travaillait aux champs de son père et se plaignait auprès de moi d'être un travailleur « bénévole ».

Le principe de la division est que chacun des fils obtienne, dans sa part d'héritage, une maison dans laquelle il puisse s'installer et fonder une nouvelle exploitation. Ahmed avait déjà construit plusieurs petites maisons (une ou

13 Que de telles disputes soient effectives ou pas, cet argument m'intéresse en tant que manière de relever, en contradiction avec les discours indigènes sur l'autorité patriarcale, la complexité des relations de pouvoir et l'imbrication des logiques des pouvoirs et des tensions au sein de l'exploitation (Mulet 2018 : 94). Dans le cas que je présente ici, le pouvoir de la dispute de Tuda et les risques de rupture sont d'autant plus importants qu'elle est une cousine germaine de Yussef et que ses parents sont voisins, proches des Utena et respectés dans le village. 
deux pièces) sur des terres lui appartenant. Utilisées dans le cadre de la production agricole, elles étaient selon lui destinées à ses fils qui en deviendraient propriétaires au moment de la division. ${ }^{14}$ Dans ce contexte, « la maison de Yussef », ainsi qu'elle était considérée, pouvait également être incluse dans le patrimoine collectif, en tant que part attribuable à l'un des fils d'Ahmed au moment du partage de ce patrimoine. La notion de propriété individuelle, cependant, était bien mise en avant lorsque je m'intéressais aux biens des membres du groupe domestique. Un jour que j'étais avec Yussef et Mustafa qui labouraient les champs qui entourent la maison de Yussef :

« Ils labourent avec la mule et l'âne de chez Said Utena. Je demande où est Said : à la maison [chez lui]. Ils s'arrêtent juste pour aller manger. On monte vers la maison [de Yussef]. Là où on marche, c'est un champ. Je demande à qui c'est. Yussef me dit que c'est à [un villageois], mais que là et là, c'est à lui [winu : le mien]. Mustafa est à côté de nous ». [extrait de mon journal de terrain, 2011]

Si je précisais dans mes notes que Mustafa était à côté de nous, c'est que j'ai été surpris de l'assurance avec laquelle son frère me déclarait être propriétaire de ces champs. Car, dans le même temps, leur père me disait être propriétaire de toute la surface cultivée par le groupe. En 2013, j'apprenais que Yussef avait acheté, profitant d'un prêt monétaire obtenu grâce à son statut professionnel, un terrain en ville pour que ses frères y construisent une maison possédée collectivement. Peu de temps après, l'aîné m’apprenait également qu'il possédait trois champs à titre personnel : «ils sont à lui, pas à toute la famille, il les a achetés », notais-je dans mon journal. Quant à Brahim, un jour que j'étais avec lui en voiture, il avait pris soin de me montrer la carte grise du véhicule afin que je puisse voir qu'il était bien enregistré à son nom.

Cette tension entre «le mien » et «le nôtre » semble structurante de la construction du groupe domestique. L'interdépendance et la collaboration constituent en effet le contexte de l'acquisition par chacun de ces hommes de patrimoine et de capitaux, ainsi que de leurs statuts personnels. Pour les aînés, quitter l'exploitation reviendrait à renoncer à ce capital collectif et aux prestations entre membres du groupe qui actualisent le groupe. Pour le père, ne pas laisser de marge de manœuvre à ses aînés constituerait un risque : les voir rompre avec le groupe qui devrait alors se passer de leur force de travail, de leurs revenus et des capitaux que ceux-ci possèdent en propre (permis de

14 Le décalage entre construction et habitat des maisons peut être considéré comme une stratégie de l'indivision par la mise en place des moyens d'une potentielle prise de distance. De plus, ainsi, chacun des fils voit que le groupe investit dans une « bonne » division en vue de laquelle il a intérêt à participer à l'accumulation collective de patrimoine. 
conduire, diplôme, capital linguistique, par exemple). Si Ahmed disait avoir un droit de regard sur l'argent de ses fils, c'est que ceux-ci jouaient ce jeu de l'indivision par laquelle ils semblaient connaître une ascension sociale. Ainsi, il m'expliquait en entretien qu'il était préférable de diviser avec un fils qui en ferait la demande, car une telle requête signifierait que ce dernier ne serait plus gêné devant son père, c'est-à-dire ne respecterait plus son autorité. Dans le cas des Utena, où la fratrie masculine est nombreuse, l'autorité d'Ahmed repose aussi sur l'équilibre permanent des forces de fission et forces de fusion, par un jeu facilité par l'importance du foncier possédé au village.

En 2014, son fils Mustafa me montrait la maison qu'il était en train de construire près de celle de son frère Yussef, m'expliquant qu'il ne souhaitait cependant pas y habiter pour rester chez son père. ${ }^{15}$ Deux ans plus tard, il n'y habitait toujours pas, tandis que son frère Brahim avait aussi commencé la construction de sa propre maison, juste à côté. Lors de ce séjour, alors que je demandais à Ahmed s'ils avaient divisé, un parent de ce dernier, qui était avec nous, m'expliqua en riant sa réponse négative : «il serre !» [izier], manière de sous-entendre qu'Ahmed travaillait à la persévérance d'une indivision qui n'allait pas de soi.

J'ai analysé ailleurs une division programmée par l'aîné (le père était décédé depuis plus de dix ans) qui m'expliquait sa fatigue à être «le patron » et le risque de voir les frères se fâcher suite aux disputes (potentielles) de leurs femmes cohabitantes (Mulet 2018). L'analyse du partage effectif du patrimoine (et notamment des maisons dans le village) permettait d'observer une forme de rupture avec un frère (célibataire) migrant qui n'envoyait plus d'argent à l'aîné, ainsi qu'avec deux sœurs célibataires. Les trois, ainsi que leur mère, ont alors quitté la maison héritée du père, qui a été attribuée à l'aîné. La décohabitation caractérise aussi l'histoire de l'exploitation d'Ahmed Utena, qui met en avant auprès de l'ethnographe l'absence de rupture au sein du groupe. L'argument de la dispute des femmes, évoqué dans ces deux cas, est une manière d'aborder la question de la cohésion du groupe domestique et l'autorité en son sein. La décohabitation de l'aîné d'Ahmed Utena et de sa femme peut être considérée comme un moyen de « continuer à aller ensemble » en créant de la distance au sein de l'exploitation, plutôt qu'un moyen de la rupture entre certains de ses membres. Cette prise de distance, cependant, se fait au risque de voir l'aîné développer sa propre production agricole sur des terres qu'il présente comme étant les siennes. C'est par de telles pratiques que les Utena, comme les Hasima, produisent des unités domestiques particulières, des configurations spatiales, économiques et sociales qui évoluent et s'imbriquent au fil des modifications des tensions qui les traversent.

15 Ce dernier l'invitait cependant, un jour que nous en discutions tous les trois, à y habiter avec sa femme et leur enfant. 


\section{CONCLUSION}

La mise en regard de ces exploitations permet de relever les conditions de leur cohésion et de l'autorité d'un homme en leur sein, c'est-à-dire les conditions de possibilité de discours indigènes qui confirment les modèles savants de l'organisation sociale locale. J'ai questionné deux postulats des théories de la communauté au Maghreb (existence d'unités domestiques définies et d'une autorité de type « patriarcal » en leur sein) à partir de l'observation de cas où la cohésion et l'autorité sont mis en discours par des hommes - pères et aînés - et, dans une certaine mesure, en pratique. Cette analyse des pratiques de ceux qui semblent dire le modèle en permet la critique : l'autorité d'un homme et le groupe sur lequel elle s'exerce ne sont pas structuralement ou culturellement donnés mais émergent, s'actualisent, se transforment et sont mis en péril dans le cadre des pratiques de celles et ceux qui font (et défont) ces groupes. Questionner ainsi la construction des groupes domestiques, c'est-àdire leur mise en forme (et donc leurs formes), nécessite d'interroger les unités mobilisées pour l'analyse ethnographique. En centrant l'analyse sur la notion d'exploitation considérée comme une configuration spatiale, sociale et économique, j'ai voulu privilégier l'approche configurationnelle développée à propos d'autres régions du monde et traiter de la polymorphie des unités domestiques en jouant la pluralité des perspectives ainsi permises.

Ces exploitations constituent bien des unités domestiques dans la mesure où j'ai pu observer une certaine cohésion du groupe ainsi défini, caractérisée notamment par l'autorité qu'exerce un homme en leur sein. Un certain nombre de conditions sont réunies pour cela. Elles sont, plus exactement, construites par les membres des exploitations qui en travaillent les aspects sociaux, économiques et spatiaux. Par exemple, dans le cas des Hasima la succession unique de Lhsen, seul fils de la fratrie, est également mise en ouvre par la construction d'une maison «de femmes » en partie autonome au sein du groupe qui exploite le patrimoine foncier agricole. Cette même problématique des jeux sur la distance, de mise en tension entre cohésion et autonomisation, entre collectivisation et individualisation, a structuré l'analyse de l'exploitation des Utena et de l'autorité du père. Considérer l'exploitation comme une unité n'équivaut pas à faire fi des tendances à la pluralisation résidentielle et budgétaire. Au contraire, il s'agit d'observer les jeux qui permettent, malgré - et parfois grâce à - des ruptures en son sein, de construire l'unité en travaillant à la cohésion symbolisée notamment par l'autorité d'un homme sur les autres membres du groupe.

Dans cette situation où le terme n'a pas d'équivalent localement, parler d'exploitation permet une certaine distance avec les termes indigènes que l'ethnographe saisit en situation et ne peut transformer en termes savants qu'au prix d'une rupture avec les situations dans lesquels ils prennent sens pour les 
co-interactants. En effet, autant qu'un objet de l'observation, l'exploitation est une unité d'analyse, une catégorie construite pour l'analyse et la description de l'organisation sociale locale. Dans un article consacré à l'écriture de l'histoire et à la représentation du passé, Paul Ricoeur avance que « les difficultés de la connaissance historique commencent avec la coupure que représente l'écriture » (Ricœur 2000). La notion de coupure est également utile pour rendre compte des logiques de l'ethnographie comme forme particulière de « réduction de la complexité » (Luhmann 2010:67) ou de construction d'un sens en défigurant « un réel où tout est réel au même titre » en y insérant de la perspective (Merleau-Ponty 1955 : 16) et en construisant des types selon certains systèmes de pertinence (Schütz 2007). Cette perspective permet de questionner la constitution des cadres de l'observation et de l'analyse ethnographique : selon quels schèmes la complexité du réel observé est-elle réduite et comment sont créés des styles pour mettre de l'ordre dans le flux du réel ? En rupture avec les notions de fixité, d'homogénéité sociale ou de limitation spatiale, souvent associées aux milieux ruraux, la notion d'exploitation permet ici de développer une approche configurationnelle pour observer dans leur complexité les processus de construction d'unités domestiques dynamiques et en tension permanente : groupes de résidence, groupes tournés vers différentes « causes communes », groupes d'exploitation d'un patrimoine, ou encore groupes sur lesquels s'exercent une autorité, par exemple. Cette perspective permet de saisir comment sont construites, dans le Haut-Atlas, des «maisons ", à la fois bâtiments d'habitation, cadres d'un pan de l'économie domestique et objets de discours sur le gouvernement domestique.

\section{BIBLIOGRAPHIE}

ARAOS, Consuelo, 2016, "When family lives nearby: kinship, socioeconomic conditions and residential configurations in Santiago, Chile”, Cuadernos ISUC, 1 (1).

ARAOS, Consuelo, 2019, Rapprochements : Proximité Résidentielle, Parenté Pratique et Conditions de Vie à Santiago, Chili. Paris, Ecole Normale Supérieure de Paris, thèse de doctorat. ARISTOTE, 1962, La Politique. Paris, Vrin.

AUGUSTINS, Georges, 1977, «Reproduction sociale et changement social : l'exemple des Baronnies », Revue Française de Sociologie, 18-3 : 465-484.

AUGUSTINS, Georges, 1998, « La perpétuation des groupes domestiques : un essai de formalisation », L'Homme, $148:$ 15-45.

BENDER, Donald R., 1967, "A refinement of the concept of household: families, co-residence, and domestic functions", American Anthropologist, nouvelle série, 69 (5) : 493-504. 
BERQUE, Jacques, 1954, Les Seksawa : Recherches sur les Structures Sociales du Haut-Atlas Occidental. Paris, Presses Universitaires de France.

BESSIÈRE, Céline, 2010, De Génération en Génération : Arrangements de Famille dans les Entreprises Viticoles de Cognac. Paris, Raisons d'Agir.

BILLAUD, Jean-Paul, 2009, « La sociologie rurale et la question territoriale : de l'évitement à la réhabilitation », Etudes Rurales, 2009/1, 183 : 113-128.

BOURDIEU, Pierre, 1962, «Célibat et condition paysanne », Etudes Rurales, 5-6 : 32-135.

BOURDIEU, Pierre, 1993, «La famille comme catégorie réalisée », Actes de la Recherche en Sciences Sociales, $100: 32-36$.

BOURDieU, Pierre, 1998, La Domination Masculine. Paris, Seuil.

Bourdieu, Pierre, 2000 [1972], Esquisse d'Une Théorie de la Pratique. Paris, Seuil.

CARSTEN, Janet, 1995, "The substance of kinship and the heat of the hearh: feeding, personhood, and relatedness among Malays in Pulau Langkawi”, American Ethnologist, 22 (2): 223-241.

CHIVA, Isac, et Joseph GOY (dirs.), 1981, Les Baronnies des Pyrénées, tome 1 : Maisons, Mode de Vie, Société. Paris, Editions de l'EHESS.

COUlanges, Numa Denis Fustel de, 1864, La Cité Antique : Etude sur le Culte, le Droit, les Institutions de la Grèce et de Rome. Paris, Durand.

DELPHY, Christine, 2000, «Patriarcat (théorie du) », en Helena Hirata et al., Dictionnaire Critique du Féminisme. Paris, Presses Universitaires de France.

DURKHEIM, Emile, 2007, De la Division du Travail Social. Paris, Presses universitaires de France.

ELIAS, Norbert, 2003 [1970], Qu'Est-ce que la Sociologie? Paris, Pocket.

FONTAINE, Laurence, 1994, «Espaces, usages et dynamiques de la dette dans les hautes vallées dauphinoises (XVIIe-XVIIIe siècles) », Annales, Histoires, Sciences Sociales, 49 (6) : 1375-1391.

FONTAINE, Laurence, 2005, « Montagnes et migrations de travail : un essai de comparaison globale (XVe-XXe siècle) », Revue d'Histoire Moderne et Contemporaine, 52 (2) : 26-48.

GELLNER, Ernest, 2003, Les Saints de l'Atlas. Saint-Denis, Bouchène.

GOLLAC, Sibylle, 2003, "Maisonnée et cause commune : une prise en charge familiale », en Florence Weber, Séverine Gojard et Agnès Gramain (dirs.), Charges de Famille : Dépendance et Parenté dans la France Contemporaine. Paris, La Découverte, 274-311.

GOLLAC, Sybille, 2005, « Faire ses partages : patrimoine professionnel et groupe de descendance $»$, Terrain, $45: 113-124$.

HAMMOUDI, Abdellah, 2007, « Phénoménologie et ethnographie : à propos de l'habitus kabyle chez Pierre Bourdieu », L'Homme, 184 : 47-83.

JAMOUS, Raymond, 1981, Honneur et Baraka : Les Structures Sociales Traditionnelles dans le Rif. Londres et Paris, Press Syndicate of the University of Cambridge, Editions de la Maison des Sciences de l'Homme.

JOLliveT, Marcel, et Henri MENDRAS (dirs.), 1971, Les Collectivités Rurales Françaises, tome 1 : Etude Comparative de Changement Social. Paris, Armand Colin.

JOLlivet, Marcel, 1974 (dir.), Les Collectivités Rurales Françaises, tome 2 : Sociétés Paysannes ou Lutte de Classes au Village. Paris, Armand Colin.

LÉVI-STRAUSS, Claude, 1979, La Voie des Masques. Paris, Plon.

LUHMANn, Niklas, 2010, Systèmes Sociaux : Esquisse d'Une Théorie Générale. Laval, Presses de l'Université de Laval. 
MAHDI, Mohamed, 1999, Pasteurs de l'Atlas : Production Pastorale, Droit et Rituel. Casablanca, Fondation Konrad Adenauer.

MAHDI, Mohamed, 2009, « La tribu au secours du développement pastoral », Etudes Rurales, 2009/2 (184) : 133-148.

MAINE, Henry Sumner, 1889, Etudes sur l'Histoire du Droit. Paris, Ernest Thorin.

MARX, Karl, 1963, Le 18 Brumaire de Louis Bonaparte. Paris, Editions Sociales.

MEILlaSSOUX, Claude, 1975, Femmes, Greniers et Capitaux. Paris, François Maspero.

MENDRAS, Henri, 1976, Sociétés Paysannes : Eléments pour Une Théorie de la Paysannerie. Paris, Armand Colin.

MERLEAU-PONTY, Maurice, 1955, Les Aventures de la Dialectique. Paris, Gallimard.

MONTAGNE, Robert, 1930, Les Berbères et le Makhzen dans le Sud du Maroc : Essai sur la Transformation Politique des Berbères Sédentaires (Groupe Chleuh). Paris, Librairie Félix Alcan.

MONTAGNE, Robert, 1953, Naissance du Prolétariat Marocain : Enquête Collective Exécutée de 1948 à 1950. Paris, Peyronnet et Cie.

MOTTA, Eugênia, 2014, "House and economy in the favela", Vibrant - Virtual Brazilian Anthropology, 11 (1) : 118-158.

Mulet, Pascal, 2018, Des Lieux Appropriés : Economies Contemporaines du Haut-Atlas. Paris, Editions Rue d'Ulm.

PERRIN-HEREDIA, Ana, 2011 , «Faire les comptes : normes comptables, normes sociales », Genèses, 3 (84) : 69-92.

PFIRSCH, Thomas, 2009, « Proximité familiale et organisation résidentielle de la parentèle dans les élites d'une ville d'Europe du Sud : l'exemple de Naples », Articulo, Journal of Urban Research, < http://articulo.revues.org/1052 > (dernière consultation en octobre 2020).

RACHIK, Hassan, 2000, Comment Rester Nomade. Casablanca, Afrique Orient.

REDFIELD, Robert, 1989, The Little Community and Peasant Society and Culture. Chicago et Londres, The University of Chicago Press.2

RICCEUR, Paul, 2000, «L'écriture de l'histoire et la représentation du passé », Annales Histoire, Sciences Sociales, $4: 731-747$.

ROSENTAL, Paul-André, 1999, Les Sentiers Invisibles : Espace, Familles et Migrations dans la France du 19e Siècle. Paris, Editions de l'Ecole des Hautes Etudes en Sciences Sociales.

SAHLINS, Marshall, 2011, "What kinship is (part one)", Journal of the Royal Anthropological Institute, 17 (1) : 2-19.

SCHÜTZ, Alfred, 2007, Essai sur le Monde Ordinaire. Paris, Le Félin.

TCHAYANOV, Alexandre, 1990, L'Organisation de l'Economie Paysanne. Paris, Editions du Regard.

TÖNNIES, Ferdinand, 1944, Communauté et Société : Catégories Fondamentales de la Sociologie Pure. Paris, Presses Universitaires de France.

WEBER, Florence, 2002, « Pour penser la parenté contemporaine : maisonnée et parentèle, des outils de l'anthropologie », en Danièle Debordeaux et Pierre Strobel (dirs.), Les Solidarités Familiales en Questions : Entraide et Transmission. Paris, L. G. D. J., 73-106.

WEBER, Florence, 2005, Le Sang, le Nom, le Quotidien : Une Sociologie de la Parenté Pratique. Paris, Aux Lieux d'Etre. 